Journal of Low Temperature Physics, Vol. 138, Nos. 3/4, February 2005 (৫ 2005)

DOI: $10.1007 / \mathrm{s} 10909-005-2273-4$

\title{
Superfluid to Mott insulator transition in one, two, and three dimensions
}

\author{
M. Köhl, H. Moritz, T. Stöferle, C. Schori, T. Esslinger \\ Institute of Quantum Electronics \\ ETH Zürich Hönggerberg \\ CH-8093 Zürich, Switzerland
}

We have created one-, two-, and three-dimensional quantum gases and study the superfluid to Mott insulator transition. Measurements of the transition using Bragg spectroscopy show that the excitation spectra of the lowdimensional superfluids differ significantly from the three-dimensional case.

PACS numbers: 05.30.Jp, 03.75.Kk, 03.75.Lm, 73.43.Nq

\section{LOW-DIMENSIONAL QUANTUM SYSTEMS}

A low dimensional gas can be created in a trap when the confining potential restricts the motion of the particles to one or two dimensions with the other motional degrees of freedom being frozen out. Quantum mechanically this implies that the particles occupy only the ground state in the restricted directions, i.e. both, the thermal energy $k_{B} T$ and the interaction energy $\mu$ have to be much smaller than the energy level spacing. In general, low-dimensional systems exhibit increased quantum fluctuations of the phase. For a homogeneous 2D gas these phase fluctuations allow BoseEinstein condensation only at zero temperature and for a homogeneous 1D gas no Bose condensation is possible at all. For trapped low-dimensional gases the Bose-Einstein phase transition takes place at finite temperatures for both, $1 \mathrm{D}^{1}$ and $2 \mathrm{D}^{2}$, but the fluctuating phase alters the properties of the gas. Low-dimensional quantum systems exhibit a wealth of fascinating phenomena whose explanations go beyond the mean-field description ${ }^{3}$. For example, in one dimension bosons acquire fermionic properties at low densities and strong interactions, forming the so-called Tonks-Girardeau gas ${ }^{4}$. For two dimensions a Berezinskii-Kosterlitz-Thouless transition is predicted resulting in the spontaneous formation of vortices from thermal excitations ${ }^{5}$.

635 
A one-dimensional gas can be realized in a cigar shaped harmonic trapping geometry characterized by the frequencies $\omega_{\perp}^{1 D}$ in the two strongly confining axes and $\omega_{z}^{1 D}$ in the weakly confining axis. A two-dimensional trap can be realized in a disk shaped geometry with one strongly confining axis characterized by $\omega_{z}^{2 D}$ and two weakly confined directions by $\omega_{\perp}^{2 D}$. The conditions for achieving the low-dimensional quantum regime then read

$$
k_{B} T, \mu \ll \begin{cases}\hbar \omega_{\perp}^{1 D} & \text { for } 1 \mathrm{D} ; \\ \hbar \omega_{z}^{2 D} & \text { for } 2 \mathrm{D} .\end{cases}
$$

A one-dimensional trapped Bose gas in the weakly interacting regime was recently created and studied ${ }^{6}$. This experiment revealed the distinctively different excitation spectrum of a one-dimensional quantum system as compared to three dimensions. Previous studies accessed a regime, where a Bose condensate with $\mu \leq \hbar \omega_{\perp}^{1 D}$ coexisted with a three-dimensional thermal $\operatorname{cloud}^{7,8}$. Also Bose gases were created in optical lattices with significant tunnelling between the one-dimensional subsystems ${ }^{9}$. Theoretical understanding of the homogeneous one-dimensional Bose gas was pioneered by Lieb and Liniger in the $1960 \mathrm{~s}^{10}$ and recently adapted to atom traps ${ }^{11-16}$. A fundamental feature of the one-dimensional Bose gas is that its ground state energy and excitation spectrum are determined by a single parameter $\gamma$ over the whole range from weak to strong interactions. $\gamma=\frac{m g_{1 D}}{\hbar^{2} n_{1 D}}$ is the ratio between interaction energy and kinetic energy $\left(m\right.$ : atomic mass, $g_{1 D}$ : $1 \mathrm{D}$ coupling constant, $n_{1 D}: 1 \mathrm{D}$ density). The transition from weak to strong interactions can be studied by applying a lattice potential along the symmetry axis of the $1 \mathrm{D}$ gas $^{17}$.

Quasi-homogeneous two-dimensional quantum gases have been studied for several years using thin films of liquid Helium on a surface ${ }^{18}$. With ultracold alkali atoms quantum degenerate atomic gases in two dimensions have been realized in a standing wave laser field ${ }^{19}$. Moreover, the regime with $\mu \leq \hbar \omega_{z}^{2 D}$ has been studied using ${ }^{23} \mathrm{Na}$ in a disk shaped optical trap where features of two-dimensional condensate expansion were observed ${ }^{7}$. With ${ }^{133} \mathrm{Cs}$ a quantum degenerate sample has been prepared in an optical trap close to a surface ${ }^{20}$ and in extremely fast rotating condensates the twodimensional regime has also been approached ${ }^{21}$. In this paper we present experiments with Bose gases fully in the 2D regime with $k_{B} T / \hbar \omega_{z}^{2 D}<6 \times$ $10^{-3}$ and $\mu / \hbar \omega_{z}^{2 D}<0.1$. The gas is subject to a periodic potential along its weakly confined directions, which allows us to tune the gas from a weakly interacting superfluid to a strongly correlated Mott insulator. 


\section{SUPERFLUID TO MOTT INSULATOR TRANSITION}

Bosons which are localized in the minima of a periodic potential may be described by the Bose-Hubbard Hamiltonian ${ }^{22,23}$ :

$$
H=-\widetilde{J} \sum_{i, j} \hat{a}_{j}^{\dagger} \hat{a}_{i}+\sum_{i} \epsilon_{i} n_{i}+\frac{U}{2} \sum_{i} n_{i}\left(n_{i}-1\right)
$$

$\widetilde{J}$ denotes the hopping matrix element between neighboring lattice sites that determines the rate of which a particle disappears from lattice site $i$ and tunnels to the adjacent lattice site $j\left(\hat{a}_{i}\right.$ and $\hat{a}_{i}^{\dagger}$ are the annihilation and creation operators for an atom at lattice site $i$, respectively). The total tunnelling rate $J$ includes the possibly anisotropic tunnelling to all next neighboring lattice sites: $J=2\left(\widetilde{J}_{x}+\widetilde{J}_{y}+\widetilde{J}_{z}\right) \cdot \epsilon_{i}$ characterizes the inhomogeneity of the atom trap. $n_{i}$ is the occupation number of lattice site $i$ and $U$ is the onsite-interaction energy between two bosons at the same lattice site. The Bose-Hubbard Hamiltonian exhibits a quantum phase transition between the superfluid state and a Mott insulator for a critical ratio $(U / J)_{c}$ which has been observed in an optical lattice, where the periodic potential is created by standing wave laser fields ${ }^{24}$. The intensity of the laser fields determines the value of $U / J$. According to a mean-field calculation one expects $(U / J)_{c}=5.8^{22,23}$. This approximation is valid for two and three dimensions, but for one spatial dimension stronger quantum fluctuations of the phase must be taken into account ${ }^{25}$. Quantum Monte-Carlo simulations show ${ }^{26}$ that these lower the onset of the Mott insulating phase to $(U / J)_{c} \simeq 1.8$. We have recently obtained experimental evidence for these fluctuations ${ }^{17}$.
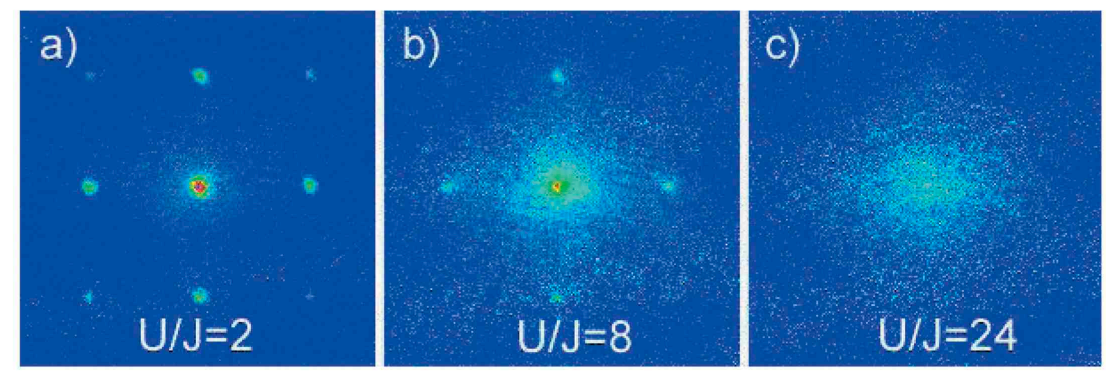

Fig. 1. Quantum phase transition from a superfluid to a Mott insulator in three dimensions. The absorption images are taken after $25 \mathrm{~ms}$ of ballistic expansion. The interference peaks in a) are separated by a momentum of $2 \hbar k$, where $k=2 \pi / \lambda$ is the wave vector of the laser creating the optical lattice. 
One signature of the phase transition can be observed by studying ballistic expansion of the atoms from the lattice. For strong tunnelling, the system is superfluid, and the wave function of an atom is delocalized over many lattice sites. The absorption image shows interference peaks with a periodicity according to the lattice vector (see Figure 1a). In contrast, for strong interactions in the Mott insulating phase the atoms are localized within a single potential well and have no definite phase relation to the next potential well. Therefore, no interference pattern develops during the expansion (see Figure 1c).

\section{EXPERIMENTAL SETUP}

\subsection{Bose condensates in an optical lattice}

In the experiment, we collect up to $2 \times 10^{9}{ }^{87} \mathrm{Rb}$ atoms in a vapor cell magneto-optical trap. After polarization gradient cooling and optical pumping into the $\left|F=2, m_{F}=2\right\rangle$ hyperfine ground state the atoms are captured in a magnetic quadrupole trap. After magnetic transport of the trapped atoms over a distance of $40 \mathrm{~cm}$ the magnetic trapping potential is converted into the harmonic and elongated potential of a QUIC trap ${ }^{27}$. Subsequently, we perform radio frequency induced evaporation of the cloud over a period of $25 \mathrm{~s}$. After evaporation we observe almost pure Bose-Einstein condensates of up to $1.5 \times 10^{5}$ atoms. Following the condensation we adiabatically change the trapping geometry to an approximately spherical symmetry with trapping frequencies of $\omega_{x}=2 \pi \times 18 \mathrm{~Hz}, \omega_{y}=2 \pi \times 20 \mathrm{~Hz}$, and $\omega_{z}=2 \pi \times 22 \mathrm{~Hz}$. This reduces the peak density by a factor 4 and allows us to load the optical lattice more uniformly.

The optical lattice is formed by three retro-reflected laser beams. Each beam is derived from a laser diode at a wavelength of $\lambda=826 \mathrm{~nm}$. At the position of the condensate the beams are circularly focused to $1 / e^{2}$-radii of $120 \mu \mathrm{m}$ ( $x$ and $y$ axes) and $105 \mu \mathrm{m}(z)$. The three beams possess mutually orthogonal polarizations and their frequencies are offset with respect to each other by several ten MHz. We stabilize the lasers to a high-finesse FabryPerot cavity, thereby reducing their line width to $\sim 10 \mathrm{kHz}$. In order to load the condensate into the ground state of the optical lattice, the intensities of the lasers are slowly increased to their final values using an exponential ramp with a time constant of $25 \mathrm{~ms}$ and a duration of $100 \mathrm{~ms}$ (see Fig. 2). The resulting optical potential depths $V_{x, y, z}$ are proportional to the laser intensities and are conveniently expressed in terms of the recoil energy $E_{R}=$ $\frac{\hbar^{2} k^{2}}{2 m}$ with $k=\frac{2 \pi}{\lambda}$ and the atomic mass $m$. 


\subsection{Preparation of low-dimensional quantum gases}

Using the optical lattice we realize one-, two-, and three-dimensional quantum gases. To prepare an array of one-dimensional tubes, two lattice axes are ramped to a fixed value $V_{\perp} \equiv V_{x}=V_{z}=30 E_{R}$. In this configuration the transverse tunnelling rates $J_{x}$ and $J_{z}$ are small and contribute only a correction of the order of $J_{x, z} / \mu \ll 1$ to the $1 \mathrm{D}$ characteristics of the individual tubes, where $\mu$ is the chemical potential of the sample. To study the superfluid to Mott insulator transition in this system we apply an additional optical lattice along the symmetry axis with a potential depth $V_{y} \ll V_{\perp}$. Upon variation of $V_{y}$ the state of the system changes from superfluid to Mott insulating. Experimentally we observe the same behavior of the system for the following preparation schemes: (i) when ramping all lattice laser beams at the same time, (ii) when first preparing a one-dimensional gas and subsequently ramping up $V_{y}$ and (iii) when creating a three-dimensional lattice first and subsequently lower $V_{y}$ to the desired value.

We prepare two-dimensional quantum gases by applying one strong lattice laser $\left(V_{z}=30 E_{R}\right)$ to freeze the atomic motion in the z-direction only. The two other laser beams are kept at lower levels with $V_{x}=V_{y}$. For the three-dimensional situation we use the same power in all three laser beams $\left(V_{x}=V_{y}=V_{z}\right)$.

\section{BRAGG SPECTROSCOPY IN AN OPTICAL LATTICE}

\subsection{Principle of the measurement}

The excitation spectrum reveals fundamental properties of the quantum state of the system. We study the excitation spectrum by two-photon Bragg spectroscopy ${ }^{17,28}$ employing amplitude modulation of the lattice potential. For the one-dimensional situation we modulate the weak axial potential, for the two-dimensional situation we modulate one of the two weak standing wave laser fields. The modulated lattice potential takes the form $V_{y}(y, t)=V_{y, 0}\left(1+A_{\text {mod }} \sin \left(2 \pi \nu_{\text {mod }} t\right)\right) \sin ^{2}(k y)$. The modulation with amplitude $A_{\text {mod }}$ and frequency $\nu_{\text {mod }}$ generates two sidebands with frequencies $\pm \nu_{\text {mod }}$ relative to the lattice laser frequency $\nu_{L}$ which define the energy $h \nu_{\text {mod }}$ of the excitation. Due to the Bragg condition atoms scattering two photons receive a momentum transfer of $0 \hbar k$ or $\pm 2 \hbar k$.

In contrast to applying a potential gradient across the lattice ${ }^{24}$, this method is not susceptible to effects like Bloch oscillations, dynamical instabilities, and Zener tunnelling which occur for low axial lattice depths. Furthermore, the excitation energy is precisely determined. 

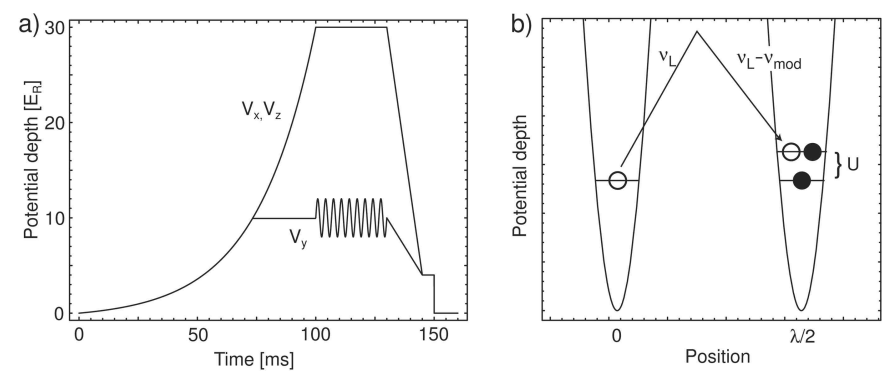

Fig. 2. a) Experimental sequence to perform Bragg spectroscopy on atoms in an optical lattice. The figure illustrates an experiment with a onedimensional gas. For a two- or three-dimensional gas the curves are accordingly different (see text). b) Scheme for resonant excitation of a Mott insulator by Bragg spectroscopy (not to scale).

After the excitation, the experimental sequence continues by ramping down the lattice potentials linearly in $15 \mathrm{~ms}$ to $V_{x, y, z}=4 E_{R}$ where the atoms are able to tunnel again in all three dimensions between the sites of the lattice. To allow for re-thermalization of the system, the atoms are kept at this lattice depth for $5 \mathrm{~ms}$. Then all optical and magnetic potentials are suddenly switched off. The switch-off time for the optical lattice is $5 \mu \mathrm{s}$ and for the magnetic trap $300 \mu \mathrm{s}$. The resulting matter wave interference pattern is detected by absorption imaging after $25 \mathrm{~ms}$ of ballistic expansion. The width of the central momentum peak is taken as a measure of how much energy has been deposited in the sample by the excitation. If the energy increase is small, the peak is well fitted by a bimodal distribution. For resonant excitation there is only a single gaussian component, reflecting that the temperature of the atoms has significantly increased. To be independent of the shape of the peak we use the full width at half maximum (FWHM) as a measure of the introduced energy. Although this underestimates small energy increases, the important resonances and features of the spectra are well shown.

We have chosen a duration $t_{\text {mod }}=30 \mathrm{~ms}$ and amplitude $A_{\text {mod }}=0.2$ of the modulation such that the resulting excitation of the condensate does not exhibit saturation effects for all measurements presented here (see Fig. 3 ). We have experimentally verified that all atoms remain in the lowest Bloch band by adiabatically switching off the lattice potentials ${ }^{9}$ after the modulation. For a thermal cloud of atoms loaded into an optical lattice we do not observe an energy increase by the modulation. 

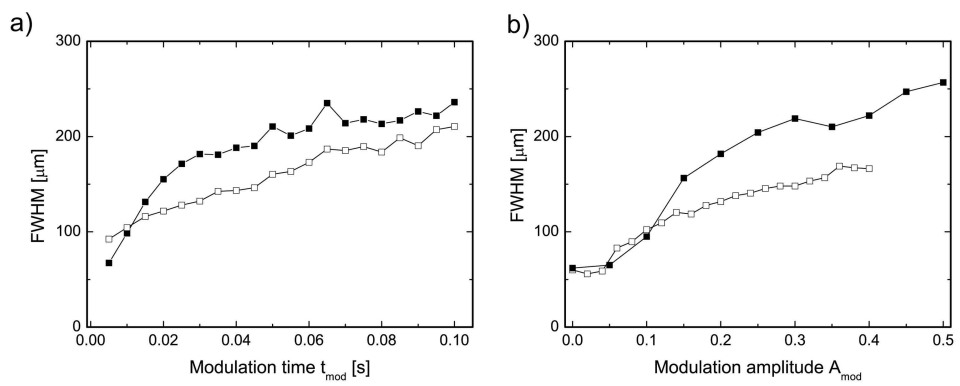

Fig. 3. Linearity of the excitation scheme for a one-dimensional gas. Filled symbols correspond to the superfluid regime, with $V_{y}=4 E_{R}$, open symbols to the Mott insulating regime with $V_{y}=10 E_{R}$. All data where taken close to the peak of the energy absorption at a modulation frequency of $\nu_{\text {mod }}=$ $1890 \mathrm{~Hz}$. a) Modulation with constant modulation index $A_{\text {mod }}=0.2$. b) Modulation with fixed duration of $0.03 \mathrm{~s}$.

\subsection{Results}

The fundamental change in the excitation spectrum when the system undergoes the quantum phase transition from a superfluid to a Mott insulator can be seen in Figure 4: The broad continuum of the superfluid $(U / J \ll 5.8)$ contrasts with the discrete spectrum of the Mott insulator $(U / J \gg 5.8)$. Figure 4 a displays the $1 \mathrm{D}$ situation with $V_{\perp}=30 E_{R}$ and includes a series of spectra for different values of $U / J$. The transition in a two-dimensional gas $\left(V_{z}=30 E_{R}\right)$ is shown in b). Finally, Figure $4 \mathrm{c}$ represents the three-dimensional case with $V_{x}=V_{y}=V_{z}$. One surprising feature is that we can excite the superfluid with our scheme at large $h \nu_{\text {mod }}$ contrary to predictions for the weakly interacting superfluid in an optical lattice formed by a single standing wave ${ }^{29}$. In our experiment strong interactions lead to a significant quantum depletion. It amounts to $\approx 50 \%$ for the 1D configuration with $U / J=2.3^{30}$. This has been studied quantitatively in a measurement of the coherence properties of the system ${ }^{17}$. Therefore this parameter may not be regarded small as in standard Bogoliubov theory and higher order excitations should be taken into account ${ }^{31}$. In combination with the broken translational invariance in the inhomogeneous trap, this could explain the non-vanishing excitation probability observed in the experiment at high energies ${ }^{32}$.

In the superfluid regime we obtain spectra which differ significantly from previous results for three dimensions ${ }^{24}$, since the superfluid excitations 

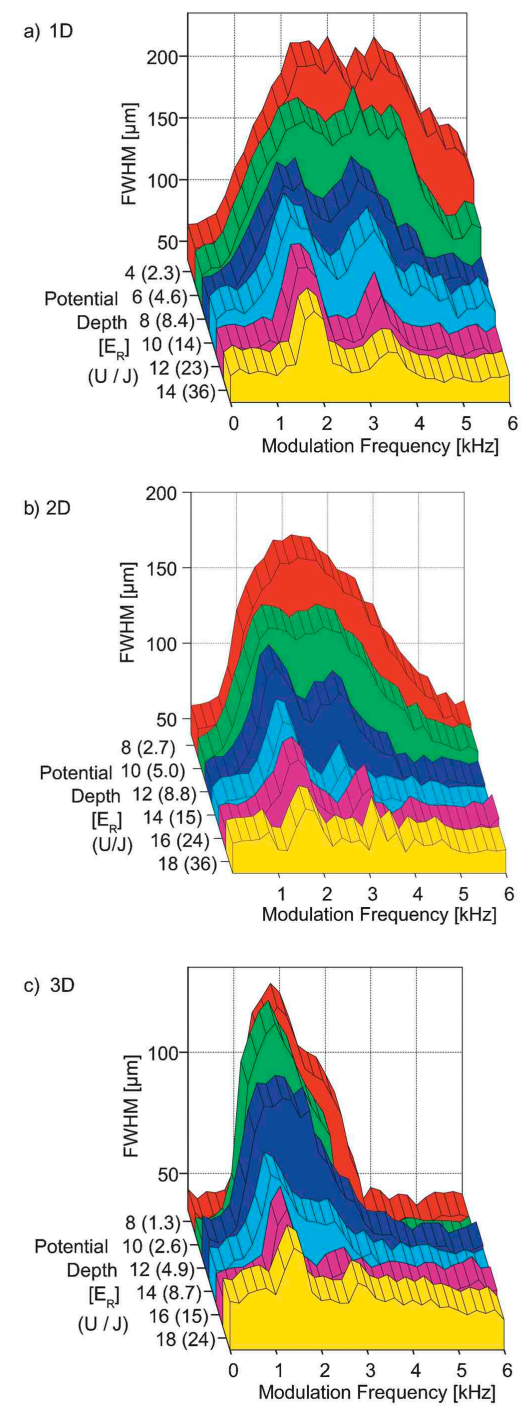

Fig. 4. The measured excitation spectra of the superfluid to Mott insulator transition. a) An array of $1 \mathrm{D}$ gases $\left(V_{x}=V_{z}=30 E_{R}\right)$. b) Superfluid to Mott insulator transition in 2D $\left(V_{z}=30 E_{R}\right)$. c) Superfluid to Mott insulator transition in 3D $\left(V_{x}=V_{y}=V_{z}\right)$. The ratios $U / J$ given in brackets are calculated numerically. The data for a) and c) are taken from reference ${ }^{17}$. 
decrease at higher energies. This decrease is rather slow for the 1D gas but becomes more pronounced for the 2D and 3D gases. Our excitation scheme does not induce dephasing that occurs when the strongly interacting condensate is accelerated near the edge of the Brillouin zone ${ }^{33}$. This might cause the broadening and the background in the tilted lattice experiments ${ }^{24}$ at high energies. The width of the superfluid spectra for the $1 \mathrm{D}$ gas is on the same order as twice the width of the lowest band for Bogoliubov excitations ${ }^{34}$.

We observe the appearance of the discrete structure, which is characteristic for the Mott insulating phase, between $U / J=4$ and $U / J=8$. Above $U / J=20$ there is no more background due to the vanishing superfluid component. However, the finite size and the inhomogeneity of the trap prohibit a sharp transition, so that the fraction of Mott insulating atoms increases gradually with $U / J$. In the Mott insulating phase we find the first resonant peak for all data sets close to the calculated value of $U$. A second peak appears at $(1.91 \pm 0.04)$ times the energy of the first resonance. We attribute this resonance to defects where lattice sites with $n=1$ atom next to sites with $n=2$ atoms are being excited. For the 1D and 2D system (Fig. 4a and b) a much weaker resonance appears at $(2.60 \pm 0.05)$ times the energy of the first resonance which could indicate higher order processes of two atoms tunnelling simultaneously.

\section{CONCLUSION}

We have studied the superfluid to Mott insulator transition in one, two, and three spatial dimensions. The excitation spectra were obtained by employing Bragg spectroscopy. We find the spectra for the low-dimensional superfluid to differ significantly from the three-dimensional case.

\section{REFERENCES}

1. W. Ketterle and N.J. van Druten, Phys. Rev. A 54, 61 (1996).

2. D. S. Petrov, M. Holzmann, and G.V. Shlyapnikov, Phys. Rev. Lett. 84, 2251 (2000).

3. T. Giamarchi, Quantum Physics in One Dimension, Oxford 2004.

4. M. Girardeau, J. Math. Phys. 1, 516 (1960).

5. V. L. Berezinskii, Sov. Phys. JETP 32493 (1971) and Sov. Phys. JETP 34610 (1972); J. M. Kosterlitz and D. J. Thouless J. Phys. C 61181 (1973) and J. Phys. C. 7, 1046 (1974).

6. H. Moritz, T. Stöferle, M. Köhl, T. Esslinger, Phys. Rev. Lett. 91, 250402 (2003).

7. A. Görlitz et al., Phys. Rev. Lett. 87, 130402 (2001). 
8. F. Schreck et al., Phys. Rev. Lett. 87, 080403 (2001).

9. M. Greiner, I. Bloch, O. Mandel, T.W. Hänsch, and T. Esslinger, Phys. Rev. Lett. 87, 160405 (2001).

10. E. H. Lieb and W. Liniger, Phys. Rev. 130, 1605 (1963); E. H. Lieb, Phys. Rev. 130, 1616 (1963).

11. M. Olshanii, Phys. Rev. Lett. 81, 938 (1998); V.Dunjko, V. Lorent, M. Olshanii, Phys. Rev. Lett. 86, 5413 (2001).

12. T.-L. Ho and M. Ma, J. Low Temp. Phys. 115, 61 (1999).

13. D.S. Petrov, G.V. Shlyapnikov, J.T.M. Walraven, Phys. Rev. Lett. 85, 3745 (2000).

14. Yu. Kagan, N. V. Prokofev, and B. V. Svistunov, Phys. Rev. A 61, 045601 (2000).

15. M. D. Girardeau, E. M. Wright, and J. M. Triscari, Phys. Rev. A 63, 033601 (2001).

16. C. Menotti and S. Stringari, Phys. Rev. A. 66, 043610 (2002).

17. T. Stöferle, H. Moritz, C. Schori, M. Köhl, T. Esslinger, Phys. Rev. Lett. 92, 130403 (2004).

18. A. I. Safonov, S. A. Vasilyev, I. S. Yasnikov, I. I. Lukashevich, and S. Jaakkola, Phys. Rev. Lett. 81, 4545 (1998).

19. C. Orzel, A. K. Tuchman, M. L. Fenselau, M. Yasuda, M. A. Kasevich, Science 291, 2386 (2001).

20. D. Rychtarik, B. Engeser, H.-C. Nägerl, and R. Grimm, e-print condmat/0309536 (2003).

21. V. Schweikhard, I. Coddington, P. Engels, V. P. Mogendor, and E. A. Cornell, Phys. Rev. Lett. 92, 040404 (2004).

22. M. P. A. Fischer, P.B. Weichmann, G. Grinstein, D.S. Fisher, Phys. Rev. B 40, 546 (1989).

23. D. Jaksch et al., Phys. Rev. Lett. 81, 3108 (1998).

24. M. Greiner et al., Nature 415, 39 (2002).

25. T. D. Kühner, H. Monien, Phys. Rev. B 58, 14741 (1998).

26. G. G. Batrouni et al., Phys. Rev. Lett. 89, 117203 (2002).

27. T. Esslinger, I. Bloch, T.W. Hänsch, Phys. Rev. A 58, 2664 (1998).

28. J. Stenger et al., Phys. Rev. Lett. 82, 4569 (1999).

29. C. Menotti, M. Krämer, L.Pitaevskii, S. Stringari, Phys. Rev. A 67, 053609 (2003).

30. M. Krämer, C. Menotti, L. Pitaevskii, S. Stringari, Eur. Phys. J. D 27, 247 (2003).

31. N.M. Hugenholtz, D. Pines, Phys. Rev. 116, 489 (1959).

32. H. P. Büchler, G. Blatter, e-print cond-mat/0312526 (2003).

33. J. C. Bronski, L.D. Carr, B. Deconinck, J.N. Kutz, Phys. Rev. Lett. 86, 1402 (2001)

34. M. Krämer, private communication.

35. Acknowledgements: This work was supported by ETH and SNF. T.E. would like to acknowledge the hospitality of the Aspen Center for Physics during the workshop "Exploring the Interface Between Cold Atoms and Condensed Matter Physics: From Strong Correlation To Entanglement". 